J. Nonlinear Var. Anal. 3 (2019), No. 2, pp. 115-126

Available online at http://jnva.biemdas.com

https://doi.org/10.23952/jnva.3.2019.2.01

\title{
ITERATIVE METHODS FOR PARALLEL CONVEX OPTIMIZATION WITH FIXED POINT CONSTRAINTS
}

\author{
KAITO SAKURAI ${ }^{1}$, TAKAYUKI JIMBA ${ }^{2}$, HIDEAKI IIDUKA ${ }^{1, *}$ \\ ${ }^{1}$ Department of Computer Science, Meiji University, Tama-ku, Kawasaki, Kanagawa 214-8571, Japan \\ ${ }^{2}$ Token Pocket Inc., 3-30-6 Soshigaya, Setagaya-ku, Tokyo 157-0072 Japan \\ Dedicated to Professor Wataru Takahashi on the occasion of his 75th birthday
}

\begin{abstract}
We consider the problem of minimizing the sum of convex functions over the intersection of fixed point sets of nonexpansive mappings. Two parallel optimization methods are investigated for solving this problem. One of the two methods is based on the Krasnosel'skiü-Mann fixed point algorithm, and the other one is based on the Halpern fixed point algorithm. We provide their convergence analyses under certain assumptions.
\end{abstract}

Keywords. Fixed point; Halpern algorithm; Krasnoselskiı̌-Mann algorithm; Parallel algorithm; Proximal point algorithm.

2010 Mathematics Subject Classification. 49M37, 65K05, 90C25.

\section{INTRODUCTION}

In this paper, we consider the problem of minimizing the sum of convex functions over the intersection of fixed point sets of nonexpansive mappings defined on a real Hilbert space [8, Problem 2.1]. Applications of the problem are, for example, optimization over generalized convex feasible set [8, 19], network resource allocation [4, 9, 10], and optimal control [11]. In relation to this problem, decentralized optimization methods have been presented $[4,10]$ for the case that the objective functions of the problem are smooth and convex, and subgradient methods have been devised [5, 7] for the case that the objective functions are convex but nonsmooth. Moreover, incremental proximal point algorithms [8] have been proposed for nonsmooth convex optimization.

In this paper, we present two parallel optimization methods for solving the problem. One of the two parallel optimization methods is based on the Krasnosel'skiř-Mann fixed point algorithm [12, 14] and the proximal point algorithm. The other one is based on the Halpern fixed point algorithm $[3,18]$ and the proximal point algorithm. We give the convergence analysis showing that there exists a subsequence of the sequence generated by the Krasnosel'skiü-Mann type algorithm which weakly converges to a solution of the problem under certain assumptions (Theorem 3.1). We also show that, if one of the convex functions is strongly convex, then the whole sequence generated by the Krasnosel'skiı-Mann type algorithm strongly converges to the unique solution (Theorem 3.1). We also give the convergence analysis showing

\footnotetext{
${ }^{*}$ Corresponding author.

E-mail addresses: sakurai.kaito08@gmail.com (K. Sakurai), yudetamago.orz@ tokenpocket.jp (T. Jimba), iiduka@cs.meiji. ac.jp (H. Iiduka).

Received December 11, 2018; Accepted April 16, 2019
}

(c)2019 Journal of Nonlinear and Variational Analysis 
that any weak sequential cluster point of the sequence generated by the Halpern type algorithm belongs to the solution set of the problem (Theorem 3.2).

\section{MAThematicAl PRELiminaries}

2.1. Notation and definitions. Let $H$ be a real Hilbert space with inner product $\langle\cdot, \cdot\rangle$ and its induced norm $\|\cdot\|$, and let $\mathbb{R}$ be the set of all real numbers and let $\mathbb{N}$ be the set of all positive integers including zero. The identity mapping on $H$ is denoted by Id. Let $\left(x_{n}\right)_{n \in \mathbb{N}}$ be a sequence in $H$. A point $x \in H$ is said to be a weak sequential cluster point of $\left(x_{n}\right)_{n \in \mathbb{N}}\left[1\right.$, Subchapters 1.7 and 2.5] if $\left(x_{n}\right)_{n \in \mathbb{N}}$ possesses a subsequence that weakly converges to $x \in H$.

A mapping $T: H \rightarrow H$ is said to be nonexpansive if $\|T(x)-T(y)\| \leq\|x-y\|(x, y \in H)$. $T$ is said to be firmly nonexpansive if

$$
\|T(x)-T(y)\|^{2}+\|(\operatorname{Id}-T)(x)-(\operatorname{Id}-T)(y)\|^{2} \leq\|x-y\|^{2} \quad(x, y \in H) .
$$

Let $\operatorname{Fix}(T):=\{x \in H: T(x)=x\}$ be the fixed point set of a mapping $T: H \rightarrow H$. The metric projection $P_{C}$ onto a nonempty, closed convex subset $C$ of $H$ is firmly nonexpansive with $\operatorname{Fix}\left(P_{C}\right)=C$ [1, Proposition 4.8, (4.8)].

Let $f: H \rightarrow(-\infty, \infty]$ be proper, lower semicontinuous, and convex. Then, the proximity operator of $f$ [1, Definition 12.23], [16], denoted by $\operatorname{Prox}_{f}$, maps every $x \in H$ to the unique minimizer of $f(\cdot)+$ $(1 / 2)\|x-\cdot\|^{2} ;$ i.e.,

$$
\operatorname{Prox}_{f}(x)=\underset{y \in H}{\operatorname{argmin}}\left[f(y)+\frac{1}{2}\|x-y\|^{2}\right] \quad(x \in H) .
$$

The uniqueness and existence of $\operatorname{Prox}_{f}(x)$ are guaranteed for all $x \in H$ [1, Definition 12.23], [15]. Let $\operatorname{dom}(f):=\{x \in H: f(x)<\infty\}$ be the domain of a function $f: H \rightarrow(-\infty, \infty]$. A function $f: H \rightarrow \mathbb{R}$ is said to be strictly convex [1, Definition 8.6] if, for all $x, y \in H$ and for all $\alpha \in(0,1), x \neq y$ implies $f(\alpha x+(1-\alpha) y)<\alpha f(x)+(1-\alpha) f(y) . f$ is strongly convex with constant $\beta$ [1, Definition 10.5] if there exists $\beta>0$ such that, for all $x, y \in H$ and for all $\alpha \in(0,1)$,

$$
f(\alpha x+(1-\alpha) y)+\frac{\beta}{2} \alpha(1-\alpha)\|x-y\|^{2} \leq \alpha f(x)+(1-\alpha) f(y) .
$$

2.2. Main problem. We will consider the following problem.

Problem 2.1. [8, Problem 2.1] Let $\mathscr{I}:=\{1,2, \ldots, I\}$. Suppose that

(A1) $T_{i}: H \rightarrow H(i \in \mathscr{I})$ is firmly nonexpansive with $\bigcap_{i \in \mathscr{I}} \operatorname{Fix}\left(T_{i}\right) \neq \emptyset$;

(A2) $f_{i}: H \rightarrow \mathbb{R}(i \in \mathscr{I})$ is continuous and convex with $\operatorname{dom}\left(f_{i}\right)=H$, and $\operatorname{Prox}_{f_{i}}$ can be computed efficiently.

Then

$$
\operatorname{minimize} f(x):=\sum_{i \in \mathscr{I}} f_{i}(x) \text { subject to } x \in X:=\bigcap_{i \in \mathscr{I}} \operatorname{Fix}\left(T_{i}\right)
$$

Problem 2.1 includes applications such as optimization over generalized convex feasible sets [8, 19], network resource allocation $[4,9,10]$, and optimal control [11]. 


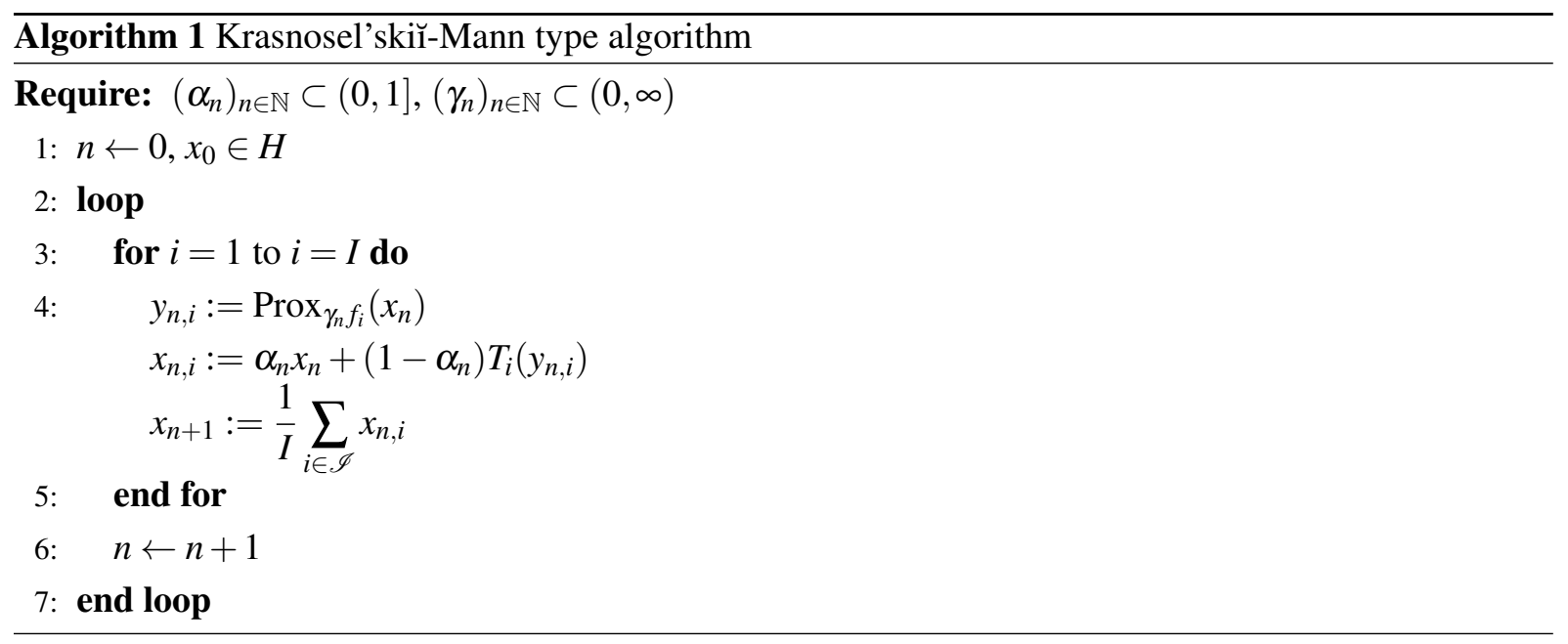

\section{Proposed Parallel Algorithms}

3.1. Krasnosel'skiî-Mann type algorithm. The algorithm is as follows.

Let us consider a network system with $I$ users. We assume that user $i$ has its own private objective function $f_{i}$ and mapping $T_{i}$ and tries to minimize $f_{i}$ over $\operatorname{Fix}\left(T_{i}\right)$. Moreover, we assume that each user can communicate with other users. Then, at iteration $n$, each user can have commonly $x_{n}$. Since user $i$ has its own objective function $f_{i}$, it computes $y_{n, i}:=\operatorname{Prox} \gamma_{n} f_{i}\left(x_{n}\right)$. Moreover, user $i$ has its own constraint set Fix $\left(T_{i}\right)$, with which it tries to find a fixed point of $T_{i}$ by using the Krasnosel'skiu-Mann fixed point algorithm $[12,14]$, i.e.,

$$
x_{n, i}:=\alpha_{n} x_{n}+\left(1-\alpha_{n}\right) T_{i}\left(y_{n, i}\right) .
$$

Since the users can communicate with each other, user $i$ can receive all $x_{n, i}$, and hence, user $i$ can compute $x_{n+1}:=(1 / I) \sum_{i \in \mathscr{I}} x_{n, i}$.

We assume the following:

Assumption 3.1. [8, Assumptions 4.1 and 4.2] The sequence $\left(y_{n, i}\right)_{n \in \mathbb{N}}(i \in \mathscr{I})$ in Algorithm 1 is bounded. Moreover, the sequences $\left(\alpha_{n}\right)_{n \in \mathbb{N}}$ and $\left(\gamma_{n}\right)_{n \in \mathbb{N}}$ satisfy the following conditions:

$$
\text { (C1) } 0<\liminf _{n \rightarrow \infty} \alpha_{n} \leq \limsup _{n \rightarrow \infty} \alpha_{n}<1 \text {, (C2) } \lim _{n \rightarrow \infty} \gamma_{n}=0 \text {, (C3) } \sum_{n=0}^{\infty} \gamma_{n}=\infty \text {. }
$$

See [8, Corollary 4.1] for an example of Algorithm 1 satisfying Assumption 3.1.

The following theorem is the convergence analysis of Algorithm 1.

Theorem 3.1. Consider Problem 2.1 and suppose that Assumption 3.1 holds. Then, there exists a subsequence of $\left(x_{n}\right)_{n \in \mathbb{N}}$ generated by Algorithm 1 which weakly converges to a solution of Problem 2.1. In addition, the sequence $\left(x_{n}\right)_{n \in \mathbb{N}}$ strongly converges to the unique solution of Problem 2.1 if one of the following holds:

(i) One $f_{i}$ is strongly convex;

(ii) $H$ is finite-dimensional, and one $f_{i}$ is strictly convex.

3.1.1. Proof of Theorem 3.1. We can prove Theorem 3.1 using the proofs of [4, Theorem 4.1], [6, Theorem 3.2], and [8, Theorem 4.1]. We first prove the following useful lemma. 
Lemma 3.1. The sequence $\left(x_{n}\right)_{n \in \mathbb{N}}$ generated by Algorithm 1 satisfies that, for all $x \in X$ and for all $n \in \mathbb{N}$,

$$
\begin{aligned}
\left\|x_{n+1}-x\right\|^{2} \leq & \left\|x_{n}-x\right\|^{2}-\frac{1}{I}\left(1-\alpha_{n}\right) \sum_{i \in \mathscr{I}}\left\{\left\|x_{n}-y_{n, i}\right\|^{2}+\left\|y_{n, i}-T_{i}\left(y_{n, i}\right)\right\|^{2}\right\} \\
& +\frac{2}{I}\left(1-\alpha_{n}\right) \gamma_{n} \sum_{i \in \mathscr{I}}\left[f_{i}(x)-f_{i}\left(y_{n, i}\right)\right] .
\end{aligned}
$$

Proof. The definition of $x_{n, i}$ and the convexity of $\|\cdot\|^{2}$ ensure that, for all $x \in X$, for all $n \in \mathbb{N}$, and for all $i \in \mathscr{I}$,

$$
\left\|x_{n, i}-x\right\|^{2}=\left\|\alpha_{n}\left(x_{n}-x\right)+\left(1-\alpha_{n}\right)\left(T_{i}\left(y_{n, i}\right)-x\right)\right\|^{2} \leq \alpha_{n}\left\|x_{n}-x\right\|^{2}+\left(1-\alpha_{n}\right)\left\|T_{i}\left(y_{n, i}\right)-x\right\|^{2},
$$

which, together with (A1), implies that

$$
\begin{aligned}
\left\|x_{n, i}-x\right\|^{2} & \leq \alpha_{n}\left\|x_{n}-x\right\|^{2}+\left(1-\alpha_{n}\right)\left\{\left\|y_{n, i}-x\right\|^{2}-\left\|y_{n, i}-T_{i}\left(y_{n, i}\right)\right\|^{2}\right\} \\
& =\alpha_{n}\left\|x_{n}-x\right\|^{2}+\left(1-\alpha_{n}\right)\left\|y_{n, i}-x\right\|^{2}-\left(1-\alpha_{n}\right)\left\|y_{n, i}-T_{i}\left(y_{n, i}\right)\right\|^{2} .
\end{aligned}
$$

From [1, Proposition 12.26] and the definition of $y_{n, i}$, for all $x \in X$, for all $n \in \mathbb{N}$, and for all $i \in \mathscr{I}$,

$$
\left\langle x-y_{n, i}, x_{n}-y_{n, i}\right\rangle \leq \gamma_{n}\left(f_{i}(x)-f_{i}\left(y_{n, i}\right)\right) .
$$

Since $\langle x, y\rangle=(1 / 2)\left(\|x\|^{2}+\|y\|^{2}-\|x-y\|^{2}\right)(x, y \in H)$ holds, we have that

$$
\left\langle x-y_{n, i}, x_{n}-y_{n, i}\right\rangle=\frac{1}{2}\left\{\left\|x-y_{n, i}\right\|^{2}+\left\|x_{n}-y_{n, i}\right\|^{2}-\left\|x-x_{n}\right\|^{2}\right\} .
$$

Hence, for all $x \in X$, for all $n \in \mathbb{N}$, and for all $i \in \mathscr{I}$,

$$
\left\|y_{n, i}-x\right\|^{2} \leq\left\|x_{n}-x\right\|^{2}-\left\|x_{n}-y_{n, i}\right\|^{2}+2 \gamma_{n}\left(f_{i}(x)-f_{i}\left(y_{n, i}\right)\right) .
$$

Accordingly, (3.1) and (3.2) guarantee that, for all $x \in X$, for all $n \in \mathbb{N}$, and for all $i \in \mathscr{I}$,

$$
\begin{aligned}
\left\|x_{n, i}-x\right\|^{2} \leq & \left\|x_{n}-x\right\|^{2}-\left(1-\alpha_{n}\right)\left\|x_{n}-y_{n, i}\right\|^{2}+2\left(1-\alpha_{n}\right) \gamma_{n}\left(f_{i}(x)-f_{i}\left(y_{n, i}\right)\right) \\
& -\left(1-\alpha_{n}\right)\left\|y_{n, i}-T_{i}\left(y_{n, i}\right)\right\|^{2} .
\end{aligned}
$$

Summing the above inequality from $i=1$ to $i=I$ and the convexity of $\|\cdot\|^{2}$ imply that, for all $x \in X$ and for all $n \in \mathbb{N}$,

$$
\begin{aligned}
I\left\|x_{n+1}-x\right\|^{2} \leq & \sum_{i \in \mathscr{I}}\left\|x_{n, i}-x\right\|^{2} \\
\leq & I\left\|x_{n}-x\right\|^{2}-\left(1-\alpha_{n}\right) \sum_{i \in \mathscr{I}}\left\{\left\|x_{n}-y_{n, i}\right\|^{2}+\left\|y_{n, i}-T_{i}\left(y_{n, i}\right)\right\|^{2}\right\} \\
& +2\left(1-\alpha_{n}\right) \gamma_{n} \sum_{i \in \mathscr{I}}\left[f_{i}(x)-f_{i}\left(y_{n, i}\right)\right],
\end{aligned}
$$

which completes the proof of Lemma 3.1.

Next, we show the following lemma indicating that Theorem 3.1 holds when $\left(x_{n}\right)_{n \in \mathbb{N}}$ in Algorithm 1 is Fejér monotone [1, Chapter 5] with respect to the solution set $X^{\star}$ of Problem 2.1.

Lemma 3.2. Suppose that Assumption 3.1 holds and that there exists $n_{0} \in \mathbb{N}$ such that, for all $x^{\star} \in X^{\star}$ and for all $n \in \mathbb{N}, n \geq n_{0}$ implies $\left\|x_{n+1}-x^{\star}\right\| \leq\left\|x_{n}-x^{\star}\right\|$. Then, the following hold:

(i) $\lim _{n \rightarrow \infty}\left\|x_{n}-y_{n, i}\right\|=0$ and $\lim _{n \rightarrow \infty}\left\|y_{n, i}-T_{i}\left(y_{n, i}\right)\right\|=0(i \in \mathscr{I})$; 
(ii) $\lim _{n \rightarrow \infty}\left\|x_{n}-x_{n, i}\right\|=0$ and $\lim _{n \rightarrow \infty}\left\|x_{n}-T_{i}\left(x_{n}\right)\right\|=0(i \in \mathscr{I})$;

(iii) $\liminf _{n \rightarrow \infty} f\left(x_{n}\right) \leq f(x)(x \in X)$;

(iv) There exists a subsequence of $\left(x_{n}\right)$ which weakly converges to a point in $X^{\star}$.

Proof. (i) From the definition of the subdifferential of $f_{i}$ (denoted by $\partial f_{i}$ ) and (A2), we have that, for all $x^{\star} \in X^{\star}$, for all $n \in \mathbb{N}$, and for all $i \in \mathscr{I}$,

$$
f_{i}\left(x^{\star}\right)-f_{i}\left(y_{n, i}\right) \leq\left\langle x^{\star}-y_{n, i}, z_{i}\right\rangle,
$$

where $z_{i} \in \partial f_{i}\left(x^{\star}\right)$. Let $x^{\star} \in X^{\star}$ be fixed arbitrarily. Since $\left(y_{n, i}\right)_{n \in \mathbb{N}}(i \in \mathscr{I})$ is bounded, there exists $N_{1} \in \mathbb{R}$ such that, for all $n \in \mathbb{N}$ and for all $i \in \mathscr{I}, f_{i}\left(x^{\star}\right)-f_{i}\left(y_{n, i}\right) \leq N_{1}$. Accordingly, Lemma 3.1 implies that, for all $n \in \mathbb{N}$ and for all $i \in \mathscr{I}$,

$$
\begin{aligned}
& \frac{1-\alpha_{n}}{I} \sum_{i \in \mathscr{I}}\left\|x_{n}-y_{n, i}\right\|^{2} \leq\left\|x_{n}-x^{\star}\right\|^{2}-\left\|x_{n+1}-x^{\star}\right\|^{2}+2 N_{1}\left(1-\alpha_{n}\right) \gamma_{n}, \\
& \frac{1-\alpha_{n}}{I} \sum_{i \in \mathscr{I}}\left\|y_{n, i}-T_{i}\left(y_{n, i}\right)\right\|^{2} \leq\left\|x_{n}-x^{\star}\right\|^{2}-\left\|x_{n+1}-x^{\star}\right\|^{2}+2 N_{1}\left(1-\alpha_{n}\right) \gamma_{n} .
\end{aligned}
$$

Since $\left(x_{n}\right)_{n \in \mathbb{N}}$ is Fejér monotone with respect to $X^{\star}$, there exists $\lim _{n \rightarrow \infty}\left\|x_{n}-x^{\star}\right\|$. Hence, (3.3), together with (C1) and (C2), yields that $\lim _{n \rightarrow \infty}\left\|x_{n}-y_{n, i}\right\|=0$ and $\lim _{n \rightarrow \infty}\left\|y_{n, i}-T_{i}\left(y_{n, i}\right)\right\|=0(i \in \mathscr{I})$.

(ii) The definition of $x_{n, i}$ and the triangle inequality mean that, for all $n \in \mathbb{N}$ and for all $i \in \mathscr{I}$,

$$
\left\|x_{n, i}-x_{n}\right\|=\left(1-\alpha_{n}\right)\left\|T_{i}\left(y_{n, i}\right)-x_{n}\right\| \leq\left\|T_{i}\left(y_{n, i}\right)-y_{n, i}\right\|+\left\|y_{n, i}-x_{n}\right\|,
$$

which, together with Lemma 3.2(i), implies that $\lim _{n \rightarrow \infty}\left\|x_{n}-x_{n, i}\right\|=0(i \in \mathscr{I})$. Moreover, for all $n \in \mathbb{N}$ and for all $i \in \mathscr{I}$,

$$
\left\|x_{n}-T_{i}\left(x_{n}\right)\right\| \leq\left\|x_{n}-y_{n, i}\right\|+\left\|y_{n, i}-T_{i}\left(y_{n, i}\right)\right\|+\left\|T_{i}\left(y_{n, i}\right)-T_{i}\left(x_{n}\right)\right\|,
$$

which, together with (A1), implies that

$$
\left\|x_{n}-T_{i}\left(x_{n}\right)\right\| \leq 2\left\|x_{n}-y_{n, i}\right\|+\left\|y_{n, i}-T_{i}\left(y_{n, i}\right)\right\| .
$$

Lemma 3.2(i) thus guarantees that $\lim _{n \rightarrow \infty}\left\|x_{n}-T_{i}\left(x_{n}\right)\right\|=0(i \in \mathscr{I})$.

(iii) Let $z_{n, i} \in \partial f_{i}\left(x_{n}\right)(n \in \mathbb{N}, i \in \mathscr{I})$. Then, (A2) and Propositions 16.14 and 16.17 in [1] imply that there exists $N_{2} \in \mathbb{R}$ such that, for all $n \in \mathbb{N}$ and for all $i \in \mathscr{I},\left\|z_{n, i}\right\| \leq N_{2}$. Accordingly, the definition of $\partial f_{i}$ guarantees that, for all $n \in \mathbb{N}$ and for all $i \in \mathscr{I}$,

$$
f_{i}\left(x_{n}\right)-f_{i}\left(y_{n, i}\right) \leq\left\langle x_{n}-y_{n, i}, z_{n, i}\right\rangle \leq N_{2}\left\|x_{n}-y_{n, i}\right\| .
$$

Hence, we have that, for all $x \in X$ and for all $n \in \mathbb{N}$,

$$
f(x)-\sum_{i \in \mathscr{I}} f_{i}\left(y_{n, i}\right)=f(x)-f\left(x_{n}\right)+\sum_{i \in \mathscr{I}}\left[f_{i}\left(x_{n}\right)-f_{i}\left(y_{n, i}\right)\right] \leq f(x)-f\left(x_{n}\right)+N_{2} \sum_{i \in \mathscr{I}}\left\|x_{n}-y_{n, i}\right\| .
$$

Lemma 3.1 thus ensures that, for all $x \in X$ and for all $n \in \mathbb{N}$,

$$
\frac{2}{I}\left(1-\alpha_{n}\right) \gamma_{n}\left\{f\left(x_{n}\right)-f(x)-N_{2} \sum_{i \in \mathscr{I}}\left\|x_{n}-y_{n, i}\right\|\right\} \leq\left\|x_{n}-x\right\|^{2}-\left\|x_{n+1}-x\right\|^{2} .
$$


Summing up (3.4) from $n=0$ to $n=m \in \mathbb{N}$ implies that

$$
\begin{aligned}
\frac{2}{I} \sum_{n=0}^{m}\left(1-\alpha_{n}\right) \gamma_{n}\left\{f\left(x_{n}\right)-f(x)-N_{2} \sum_{i \in \mathscr{I}}\left\|x_{n}-y_{n, i}\right\|\right\} & \leq\left\|x_{0}-x\right\|^{2}-\left\|x_{m+1}-x\right\|^{2} \\
& \leq\left\|x_{0}-x\right\|^{2}<\infty .
\end{aligned}
$$

Therefore, we have that, for all $x \in X$,

$$
\sum_{n=0}^{\infty}\left(1-\alpha_{n}\right) \gamma_{n}\left\{f\left(x_{n}\right)-f(x)-N_{2} \sum_{i \in \mathscr{I}}\left\|x_{n}-y_{n, i}\right\|\right\}<\infty .
$$

We shall show that, for all $x \in X$,

$$
\liminf _{n \rightarrow \infty}\left(1-\alpha_{n}\right)\left\{f\left(x_{n}\right)-f(x)-N_{2} \sum_{i \in \mathscr{I}}\left\|x_{n}-y_{n, i}\right\|\right\} \leq 0 .
$$

If (3.6) does not hold, there exist $x \in X, m_{0} \in \mathbb{N}$, and $\gamma>0$ such that, for all $n \geq m_{0}$,

$$
\left(1-\alpha_{n}\right)\left\{f\left(x_{n}\right)-f(x)-N_{2} \sum_{i \in \mathscr{I}}\left\|x_{n}-y_{n, i}\right\|\right\} \geq \gamma
$$

Then, (3.5) and (C3) guarantee that

$$
\infty=\gamma \sum_{n=m_{0}}^{\infty} \gamma_{n} \leq \sum_{n=m_{0}}^{\infty}\left(1-\alpha_{n}\right) \gamma_{n}\left\{f\left(x_{n}\right)-f(x)-N_{2} \sum_{i \in \mathscr{I}}\left\|x_{n}-y_{n, i}\right\|\right\}<\infty,
$$

which is a contradiction. Hence, (3.6) holds for all $x \in X$. Lemma 3.2(i) and (C1) thus imply that, for all $x \in X$,

$$
\liminf _{n \rightarrow \infty} f\left(x_{n}\right) \leq f(x)
$$

(iv) From Lemma 3.2(iii), there exists a subsequence $\left(x_{n_{k}}\right)_{k \in \mathbb{N}}$ of $\left(x_{n}\right)_{n \in \mathbb{N}}$ such that, for all $x \in X$,

$$
\lim _{k \rightarrow \infty} f\left(x_{n_{k}}\right)=\liminf _{n \rightarrow \infty} f\left(x_{n}\right) \leq f(x) .
$$

Since $\left(x_{n}\right)_{n \in \mathbb{N}}$ is bounded, we have $\left(x_{n_{k_{l}}}\right)_{l \in \mathbb{N}} \subset\left(x_{n_{k}}\right)_{k \in \mathbb{N}}$ which weakly converges to $x^{*} \in H$. We next show that $x^{*} \in X=\bigcap_{i \in \mathscr{I}} \operatorname{Fix}\left(T_{i}\right)$. If this assertion does not hold, there exists $i_{0} \in \mathscr{I}$ such that $x^{*} \notin \operatorname{Fix}\left(T_{i_{0}}\right)$. In this case, Opial's lemma [17, Lemma 3.1] leads to

$$
\liminf _{l \rightarrow \infty}\left\|x_{n_{k_{l}}}-x^{*}\right\|<\liminf _{l \rightarrow \infty}\left\|x_{n_{k_{l}}}-T_{i_{0}}\left(x^{*}\right)\right\|,
$$

which, together with the triangle inequality, Lemma 3.2(ii), and (A1), implies that

$$
\liminf _{l \rightarrow \infty}\left\|x_{n_{k_{l}}}-x^{*}\right\|<\lim _{l \rightarrow \infty}\left\|x_{n_{k_{l}}}-T_{i_{0}}\left(x_{n_{k_{l}}}\right)\right\|+\liminf _{l \rightarrow \infty}\left\|T_{i_{0}}\left(x_{n_{k_{l}}}\right)-T_{i_{0}}\left(x^{*}\right)\right\| \leq \liminf _{l \rightarrow \infty}\left\|x_{n_{k_{l}}}-x^{*}\right\| .
$$

This is a contradiction. Hence, we have $x^{*} \in X$. Moreover, (3.7) and (A2) ensure that, for all $x \in X$,

$$
f\left(x^{*}\right) \leq \lim _{l \rightarrow \infty} f\left(x_{n_{k_{l}}}\right)=\lim _{k \rightarrow \infty} f\left(x_{n_{k}}\right)=\liminf _{n \rightarrow \infty} f\left(x_{n}\right) \leq f(x),
$$

which implies that $x^{*} \in X^{\star}$. Let $\left(x_{n_{k_{m}}}\right)_{m \in \mathbb{N}}$ be a subsequence of $\left(x_{n_{k}}\right)_{k \in \mathbb{N}}$ which weakly converges to $x_{*} \in H$. The same discussion as in the proof of $x^{*} \in X^{\star}$ leads to $x_{*} \in X^{\star}$. If $x^{*} \neq x_{*}$ holds, then the existence of $L:=\lim _{n \rightarrow \infty}\left\|x_{n}-x^{\star}\right\|\left(x^{\star} \in X^{\star}\right)$ and Opial's lemma [17, Lemma 3.1] imply that

$$
L=\lim _{l \rightarrow \infty}\left\|x_{n_{k_{l}}}-x^{*}\right\|<\lim _{l \rightarrow \infty}\left\|x_{n_{k_{l}}}-x_{*}\right\|=\lim _{n \rightarrow \infty}\left\|x_{n}-x_{*}\right\|=\lim _{m \rightarrow \infty}\left\|x_{n_{k_{m}}}-x_{*}\right\|<\lim _{m \rightarrow \infty}\left\|x_{n_{k_{m}}}-x^{*}\right\|=L,
$$


which is a contradiction. Accordingly, any subsequence of $\left(x_{n_{k}}\right)_{k \in \mathbb{N}}$ converges weakly to $x^{*} \in X^{\star}$. This completes the proof.

We show the following lemma indicating that Theorem 3.1 holds when $\left(x_{n}\right)_{n \in \mathbb{N}}$ in Algorithm 1 is not Fejér monotone [1, Chapter 5] with respect to $X^{\star}$.

Lemma 3.3. Suppose that Assumption 3.1 holds and that, for all $n \in \mathbb{N}$, there exist $x^{\star} \in X^{\star}$ and $n_{1} \in \mathbb{N}$ such that $n_{1} \geq n$ and $\left\|x_{n_{1}+1}-x^{\star}\right\|>\left\|x_{n_{1}}-x^{\star}\right\|$. Then, there exists a subsequence $\left(x_{\tau(n)}\right)_{n \in \mathbb{N}}$ of $\left(x_{n}\right)_{n \in \mathbb{N}}$, where $\tau(n):=\max \left\{k \leq n:\left\|x_{k}-x^{\star}\right\|<\left\|x_{k+1}-x^{\star}\right\|\right\}$, which satisfies the following:

(i) $\lim _{n \rightarrow \infty}\left\|x_{\tau(n)}-y_{\tau(n), i}\right\|=0$ and $\lim _{n \rightarrow \infty}\left\|y_{\tau(n), i}-T_{i}\left(y_{\tau(n), i}\right)\right\|=0(i \in \mathscr{I})$;

(ii) $\lim _{n \rightarrow \infty}\left\|x_{\tau(n)}-x_{\tau(n), i}\right\|=0$ and $\lim _{n \rightarrow \infty}\left\|x_{\tau(n)}-T_{i}\left(x_{\tau(n)}\right)\right\|=0(i \in \mathscr{I})$;

(iii) $\limsup _{n \rightarrow \infty} f\left(x_{\tau(n)}\right) \leq f\left(x^{\star}\right)$;

(iv) There exists a subsequence of $\left(x_{\tau(n)}\right)$ which weakly converges to a point in $X^{\star}$.

Proof. Let $\Gamma_{n}:=\left\|x_{n}-x^{\star}\right\|$ for all $n \in \mathbb{N}$. Since $\left(x_{n}\right)_{n \in \mathbb{N}}$ is not Fejér monotone, there exists $\left(x_{n_{j}}\right)_{j \in \mathbb{N}} \subset$ $\left(x_{n}\right)_{n \in \mathbb{N}}$ such that, for all $j \in \mathbb{N},\left\|x_{n_{j}}-x^{\star}\right\|<\left\|x_{n_{j}+1}-x^{\star}\right\|$. In this case, Lemma 2.1 in [13] ensures that $\lim _{n \rightarrow \infty} \tau(n)=\infty$ and that there exists $m_{1} \in \mathbb{N}$ such that, for all $n \geq m_{1}, \Gamma_{\tau(n)} \leq \Gamma_{\tau(n)+1}$.

(i) From (3.3), we have that

$$
\begin{aligned}
& \frac{1-\alpha_{\tau(n)}}{I} \sum_{i \in \mathscr{I}}\left\|x_{\tau(n)}-y_{\tau(n), i}\right\|^{2} \leq 2 N_{1}\left(1-\alpha_{\tau(n)}\right) \gamma_{\tau(n)}, \\
& \frac{1-\alpha_{\tau(n)}}{I} \sum_{i \in \mathscr{I}}\left\|y_{\tau(n), i}-T_{i}\left(y_{\tau(n), i}\right)\right\|^{2} \leq 2 N_{1}\left(1-\alpha_{\tau(n)}\right) \gamma_{\tau(n)},
\end{aligned}
$$

which, together with (C1) and (C2), implies that $\lim _{n \rightarrow \infty}\left\|x_{\tau(n)}-y_{\tau(n), i}\right\|=0$ and

$$
\lim _{n \rightarrow \infty}\left\|y_{\tau(n), i}-T_{i}\left(y_{\tau(n), i}\right)\right\|=0(i \in \mathscr{I}) .
$$

Since $\left(y_{n, i}\right)_{n \in \mathbb{N}}(i \in \mathscr{I})$ is bounded, $\left(x_{\tau(n)}\right)_{n \in \mathbb{N}}$ is also bounded.

(ii) The same reasoning as in the proofs of Lemmas 3.2(ii) and 3.3(i) leads to

$$
\lim _{n \rightarrow \infty}\left\|x_{\tau(n)}-x_{\tau(n), i}\right\|=0, \lim _{n \rightarrow \infty}\left\|x_{\tau(n)}-T_{i}\left(x_{\tau(n)}\right)\right\|=0(i \in \mathscr{I}) .
$$

(iii) From (3.4) and $\Gamma_{\tau(n)} \leq \Gamma_{\tau(n)+1}\left(n \geq m_{1}\right)$, we have that, for all $n \geq m_{1}$,

$$
\frac{2}{I}\left(1-\alpha_{\tau(n)}\right) \gamma_{\tau(n)}\left\{f\left(x_{\tau(n)}\right)-f\left(x^{\star}\right)-N_{2} \sum_{i \in \mathscr{I}}\left\|x_{\tau(n)}-y_{\tau(n), i}\right\|\right\} \leq \Gamma_{\tau(n)}^{2}-\Gamma_{\tau(n)+1}^{2} \leq 0,
$$

which implies that, for all $n \geq m_{1}$,

$$
f\left(x_{\tau(n)}\right)-f\left(x^{\star}\right)-N_{2} \sum_{i \in \mathscr{I}}\left\|x_{\tau(n)}-y_{\tau(n), i}\right\| \leq 0 .
$$

Accordingly,

$$
\limsup _{n \rightarrow \infty} f\left(x_{\tau(n)}\right) \leq f\left(x^{\star}\right)+N_{2} \lim _{n \rightarrow \infty} \sum_{i \in \mathscr{I}}\left\|x_{\tau(n)}-y_{\tau(n), i}\right\|=f\left(x^{\star}\right) .
$$

(iv) From Lemma 3.3(iii), we can choose any subsequence $\left(x_{\tau\left(n_{k}\right)}\right)_{k \in \mathbb{N}}$ of $\left(x_{\tau(n)}\right)_{n \geq m_{1}}$ such that

$$
\limsup _{k \rightarrow \infty} f\left(x_{\tau\left(n_{k}\right)}\right) \leq \limsup _{n \rightarrow \infty} f\left(x_{\tau(n)}\right) .
$$


The boundedness of $\left(x_{\tau\left(n_{k}\right)}\right)_{k \in \mathbb{N}}$ guarantees the existence of $\left(x_{\tau\left(n_{k_{l}}\right)}\right)_{l \in \mathbb{N}} \subset\left(x_{\tau\left(n_{k}\right)}\right)_{k \in \mathbb{N}}$ which weakly converges to $\hat{x} \in H$. The same discussion as in the proof of Lemma 3.2(iv) leads to $\hat{x} \in X$. Lemma 3.3(iii) and (A2) thus ensure that

$$
f\left(x^{\star}\right) \leq f(\hat{x}) \leq \limsup _{l \rightarrow \infty} f\left(x_{\tau\left(n_{k_{l}}\right)}\right) \leq \limsup _{k \rightarrow \infty} f\left(x_{\tau\left(n_{k}\right)}\right) \leq \limsup _{n \rightarrow \infty} f\left(x_{\tau(n)}\right) \leq f\left(x^{\star}\right),
$$

which implies that $\hat{x} \in X^{\star}$. This completes the proof.

Theorem 3.1 and the proof of [6, Theorem 3.2] lead to the following lemma. Hence, we will omit the proof here.

Lemma 3.4. Suppose that Assumption 3.1 and one of conditions (i) and (ii) in Theorem 3.1 hold. Then, $\left(x_{n}\right)_{n \in \mathbb{N}}$ strongly converges to the unique point in $X^{\star}$.

3.2. Halpern type algorithm. The algorithm is as follows:

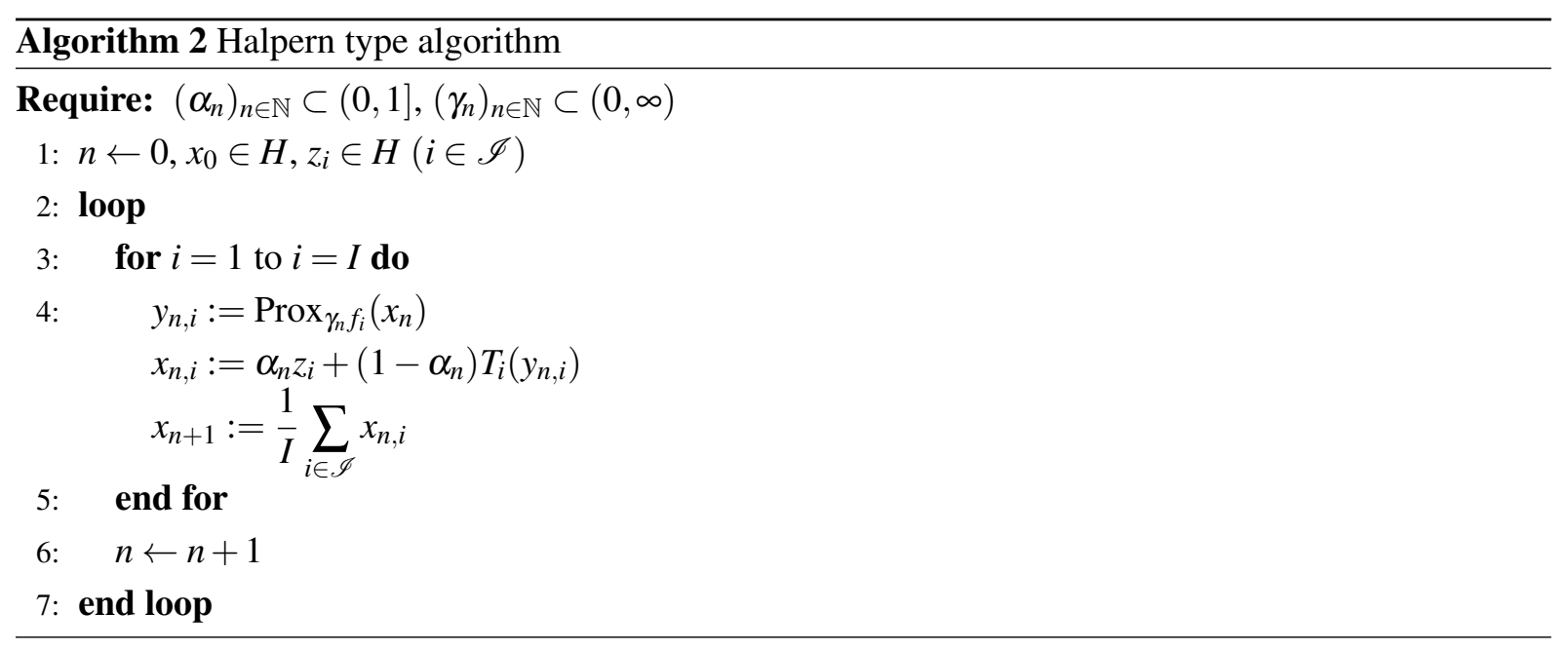

Algorithm 2 can be obtained by replacing $x_{n, i}$ in Algorithm 1 with the point computed by the Halpern fixed point algorithm $[3,18]$, i.e., $x_{n, i}:=\alpha_{n} z_{i}+\left(1-\alpha_{n}\right) T_{i}\left(y_{n, i}\right)$.

We assume the following.

Assumption 3.2. [8, Assumptions 3.1 and 3.2] The sequence $\left(y_{n, i}\right)_{n \in \mathbb{N}}(i \in \mathscr{I})$ in Algorithm 1 is bounded. Moreover, the sequences $\left(\alpha_{n}\right)_{n \in \mathbb{N}}$ and $\left(\gamma_{n}\right)_{n \in \mathbb{N}}$ converge to zero and satisfy the following conditions:

$$
\begin{aligned}
& \text { (C1) } \sum_{n=0}^{\infty} \alpha_{n}=\infty, \text { (C2) } \lim _{n \rightarrow \infty} \frac{1}{\alpha_{n+1}}\left|\frac{1}{\gamma_{n+1}}-\frac{1}{\gamma_{n}}\right|=0, \text { (C3) } \lim _{n \rightarrow \infty} \frac{\alpha_{n}}{\gamma_{n}}=0, \\
& \text { (C4) } \lim _{n \rightarrow \infty} \frac{1}{\gamma_{n+1}}\left|1-\frac{\alpha_{n}}{\alpha_{n+1}}\right|=0, \text { (C5) } \lim _{n \rightarrow \infty} \frac{1}{\alpha_{n+1}} \frac{\left|\gamma_{n+1}-\gamma_{n}\right|}{\gamma_{n+1}^{2}}=0 .
\end{aligned}
$$

See [8, Corollary 3.1] for an example of Algorithm 1 satisfying Assumption 3.2.

The following theorem is the convergence analysis of Algorithm 2.

Theorem 3.2. Consider Problem 2.1 and suppose that Assumption 3.2 holds. Then, any weak sequential cluster point of the sequence $\left(x_{n}\right)_{n \in \mathbb{N}}$ generated by Algorithm 2 belongs to the solution set of Problem 2.1. 
3.2.1. Proof of Theorem 3.2. We can prove Theorem 3.2 using the proofs of [4, Theorem 4.1] and [8, Theorem 3.1]. First, we give the following lemma.

Lemma 3.5. Suppose that Assumption 3.2 holds. Then, the following hold:

(i) $\lim _{n \rightarrow \infty}\left\|x_{n+1}-x_{n}\right\| / \gamma_{n}=0$;

(ii) $\lim _{n \rightarrow \infty}\left\|y_{n, i}-T_{i}\left(y_{n, i}\right)\right\|=0$ and $\lim _{n \rightarrow \infty}\left\|x_{n}-y_{n, i}\right\|=0(i \in \mathscr{I})$;

(iii) $\lim _{n \rightarrow \infty}\left\|x_{n}-T_{i}\left(x_{n}\right)\right\|=0(i \in \mathscr{I})$.

Proof. (i) The triangle inequality and the definition of $x_{n, i}$ imply that, for all $n \geq 1$ and for all $i \in \mathscr{I}$,

$$
\left\|x_{n, i}-x_{n-1, i}\right\| \leq\left(1-\alpha_{n}\right)\left\|T_{i}\left(y_{n, i}\right)-T_{i}\left(y_{n-1, i}\right)\right\|+\left|\alpha_{n}-\alpha_{n-1}\right|\left\|z_{i}-T_{i}\left(y_{n-1, i}\right)\right\| .
$$

The boundedness of $\left(y_{n, i}\right)_{n \in \mathbb{N}}(i \in \mathscr{I})$ and the nonexpansivity of $T_{i}$ guarantee that there exists $M_{1} \in \mathbb{R}$ such that, for all $n \geq 1$ and for all $i \in \mathscr{I},\left\|z_{i}-T_{i}\left(y_{n-1, i}\right)\right\| \leq M_{1}$. The nonexpansivity of $T_{i}$ thus ensures that, for all $n \geq 1$ and for all $i \in \mathscr{I}$,

$$
\left\|x_{n, i}-x_{n-1, i}\right\| \leq\left(1-\alpha_{n}\right)\left\|y_{n, i}-y_{n-1, i}\right\|+M_{1}\left|\alpha_{n}-\alpha_{n-1}\right| .
$$

Let $\bar{y}_{n, i}:=\operatorname{Prox} \gamma_{n} f_{i}\left(x_{n-1}\right)$ for all $n \geq 1$ and for all $i \in \mathscr{I}$. The nonexpansivity of $\operatorname{Prox} \gamma_{n} f_{i}$ and the triangle inequality imply that, for all $n \geq 1$ and for all $i \in \mathscr{I}$,

$$
\left\|y_{n, i}-y_{n-1, i}\right\| \leq\left\|y_{n, i}-\bar{y}_{n, i}\right\|+\left\|\bar{y}_{n, i}-y_{n-1, i}\right\| \leq\left\|x_{n}-x_{n-1}\right\|+\left\|\bar{y}_{n, i}-y_{n-1, i}\right\| .
$$

From [1, Proposition 12.26] and the definition of $y_{n, i}$, for all $x \in H$, for all $n \in \mathbb{N}$, and for all $i \in \mathscr{I}$,

$$
\left\langle x-y_{n, i}, \frac{x_{n}-y_{n, i}}{\gamma_{n}}\right\rangle \leq f_{i}(x)-f_{i}\left(y_{n, i}\right),
$$

which implies that $\left(x_{n}-y_{n, i}\right) / \gamma_{n} \in \partial f_{i}\left(y_{n, i}\right)$. The same discussion as for showing $\left(x_{n}-y_{n, i}\right) / \gamma_{n} \in \partial f_{i}\left(y_{n, i}\right)$ means that $\left(x_{n-1}-\bar{y}_{n, i}\right) / \gamma_{n} \in \partial f_{i}\left(\bar{y}_{n, i}\right)$. Accordingly, for all $n \geq 1$ and for all $i \in \mathscr{I}$,

$$
\left\langle y_{n-1, i}-\bar{y}_{n, i}, \frac{x_{n-1}-y_{n-1, i}}{\gamma_{n-1}}-\frac{x_{n-1}-\bar{y}_{n, i}}{\gamma_{n}}\right\rangle \geq 0
$$

which implies that

$$
\frac{1}{\gamma_{n-1} \gamma_{n}}\left\{\left(\gamma_{n}-\gamma_{n-1}\right)\left\langle y_{n-1, i}-\bar{y}_{n, i}, x_{n-1}\right\rangle-\gamma_{n}\left\|y_{n-1, i}-\bar{y}_{n, i}\right\|^{2}+\left(\gamma_{n-1}-\gamma_{n}\right)\left\langle y_{n-1, i}-\bar{y}_{n, i}, \bar{y}_{n, i}\right\rangle\right\} \geq 0 .
$$

Hence, for all $n \in \mathbb{N}$ and for all $i \in \mathscr{I}$,

$$
\left\|y_{n-1, i}-\bar{y}_{n, i}\right\|^{2} \leq \frac{\left|\gamma_{n}-\gamma_{n-1}\right|}{\gamma_{n}}\left|\left\langle y_{n-1, i}-\bar{y}_{n, i}, x_{n-1}-\bar{y}_{n, i}\right\rangle\right| .
$$

Since $\left(T_{i}\left(y_{n, i}\right)\right)_{n \in \mathbb{N}}(i \in \mathscr{I})$ is bounded, $\left(x_{n, i}\right)_{n \in \mathbb{N}}(i \in \mathscr{I})$ is also bounded. The definition of $x_{n}$ thus implies that $\left(x_{n}\right)_{n \in \mathbb{N}}$ is bounded. Accordingly, there exists $M_{2} \in \mathbb{R}$ such that, for all $n \geq 1$ and for all $i \in \mathscr{I},\left\|x_{n-1}-\bar{y}_{n, i}\right\| \leq M_{2}$. Hence, (3.10) ensures that, for all $n \in \mathbb{N}$ and for all $i \in \mathscr{I}$,

$$
\left\|y_{n-1, i}-\bar{y}_{n, i}\right\| \leq M_{2} \frac{\left|\gamma_{n}-\gamma_{n-1}\right|}{\gamma_{n}} .
$$

Inequalities (3.8), (3.9), and (3.11), together with the definition of $x_{n}$, guarantee that, for all $n \geq 1$,

$$
\left\|x_{n+1}-x_{n}\right\| \leq\left(1-\alpha_{n}\right)\left\|x_{n}-x_{n-1}\right\|+M_{1}\left|\alpha_{n}-\alpha_{n-1}\right|+M_{2} \frac{\left|\gamma_{n}-\gamma_{n-1}\right|}{\gamma_{n}} .
$$


Therefore, for all $n \geq 1$,

$$
\frac{\left\|x_{n+1}-x_{n}\right\|}{\gamma_{n}} \leq\left(1-\alpha_{n}\right) \frac{\left\|x_{n}-x_{n-1}\right\|}{\gamma_{n-1}}+M_{3}\left|\frac{1}{\gamma_{n}}-\frac{1}{\gamma_{n-1}}\right|+M_{1} \frac{\left|\alpha_{n}-\alpha_{n-1}\right|}{\gamma_{n}}+M_{2} \frac{\left|\gamma_{n}-\gamma_{n-1}\right|}{\gamma_{n}^{2}},
$$

where $M_{3}:=\sup \left\{\left\|x_{n}-x_{n-1}\right\|: n \geq 1\right\}<\infty$. From [2, Lemma 1.2] and Assumption 3.2, we have that $\lim _{n \rightarrow \infty}\left\|x_{n+1}-x_{n}\right\| / \gamma_{n}=0$.

(ii) The convexity of $\|\cdot\|^{2}$ and (A1) ensure that, for all $x \in X$, for all $n \in \mathbb{N}$, and for all $i \in \mathscr{I}$,

$$
\left\|x_{n, i}-x\right\|^{2} \leq \alpha_{n}\left\|z_{i}-x\right\|^{2}+\left(1-\alpha_{n}\right)\left\{\left\|y_{n, i}-x\right\|^{2}-\left\|y_{n, i}-T_{i}\left(y_{n, i}\right)\right\|^{2}\right\} .
$$

From [1, Proposition 12.26] and the definition of $y_{n, i}$, we have that, for all $x \in X$, for all $n \in \mathbb{N}$, and for all $i \in \mathscr{I}$,

$$
\left\langle x-y_{n, i}, x_{n}-y_{n, i}\right\rangle \leq \gamma_{n}\left[f_{i}(x)-f_{i}\left(y_{n, i}\right)\right]
$$

Accordingly,

$$
\frac{1}{2}\left\{\left\|x-y_{n, i}\right\|^{2}+\left\|x_{n}-y_{n, i}\right\|^{2}-\left\|x-x_{n}\right\|^{2}\right\} \leq \gamma_{n}\left[f_{i}(x)-f_{i}\left(y_{n, i}\right)\right],
$$

which implies that

$$
\left\|y_{n, i}-x\right\|^{2} \leq\left\|x_{n}-x\right\|^{2}-\left\|x_{n}-y_{n, i}\right\|^{2}+2 \gamma_{n}\left[f_{i}(x)-f_{i}\left(y_{n, i}\right)\right] .
$$

Let $x \in X$ be fixed arbitrarily. Then, (3.12) and (3.13) guarantee that, for all $n \in \mathbb{N}$ and for all $i \in \mathscr{I}$,

$$
\left\|x_{n, i}-x\right\|^{2} \leq M_{4} \alpha_{n}+\left\|x_{n}-x\right\|^{2}-\left\|x_{n}-y_{n, i}\right\|^{2}+2 \gamma_{n}\left[f_{i}(x)-f_{i}\left(y_{n, i}\right)\right]-\left(1-\alpha_{n}\right)\left\|y_{n, i}-T_{i}\left(y_{n, i}\right)\right\|^{2},
$$

where $M_{4}:=\max _{i \in \mathscr{I}}\left\|z_{i}-x\right\|<\infty$. Accordingly, for all $n \in \mathbb{N}$,

$$
\begin{aligned}
\left\|x_{n+1}-x\right\|^{2} \leq & \left\|x_{n}-x\right\|^{2}-\frac{1}{I} \sum_{i \in \mathscr{I}}\left\|x_{n}-y_{n, i}\right\|^{2}-\frac{1-\alpha_{n}}{I} \sum_{i \in \mathscr{I}}\left\|y_{n, i}-T_{i}\left(y_{n, i}\right)\right\|^{2} \\
& +\frac{2}{I} \gamma_{n} \sum_{i \in \mathscr{I}}\left[f_{i}(x)-f_{i}\left(y_{n, i}\right)\right]+M_{4} \alpha_{n} .
\end{aligned}
$$

Moreover, the boundedness of $\left(y_{n, i}\right)_{n \in \mathbb{N}}(i \in \mathscr{I})$ and (A2) imply that there exists $M_{5} \in \mathbb{R}$ such that, for all $n \in \mathbb{N}$ and for all $i \in \mathscr{I}, 2\left[f_{i}(x)-f_{i}\left(y_{n, i}\right)\right] \leq M_{5}$. Hence, (3.14) ensures that, for all $n \in \mathbb{N}$,

$$
\frac{1}{I} \sum_{i \in \mathscr{I}}\left\|x_{n}-y_{n, i}\right\|^{2}+\frac{1-\alpha_{n}}{I} \sum_{i \in \mathscr{I}}\left\|y_{n, i}-T_{i}\left(y_{n, i}\right)\right\|^{2} \leq\left\|x_{n}-x\right\|^{2}-\left\|x_{n+1}-x\right\|^{2}+M_{4} \alpha_{n}+M_{5} \gamma_{n} .
$$

Since Lemma 3.5(i) implies that $\lim _{n \rightarrow \infty}\left\|x_{n+1}-x_{n}\right\|=0$, we have that

$$
\begin{aligned}
\limsup _{n \rightarrow \infty}\left(\left\|x_{n}-x\right\|^{2}-\left\|x_{n+1}-x\right\|^{2}\right) & \leq \limsup _{n \rightarrow \infty}\left(\left\|x_{n}-x\right\|+\left\|x_{n+1}-x\right\|\right)\left\|x_{n+1}-x_{n}\right\| \\
& \leq 0 .
\end{aligned}
$$

Accordingly, Assumption 3.2 guarantees that

$$
\lim _{n \rightarrow \infty} \sum_{i \in \mathscr{I}}\left\|x_{n}-y_{n, i}\right\|^{2}=0, \lim _{n \rightarrow \infty} \frac{1-\alpha_{n}}{I} \sum_{i \in \mathscr{I}}\left\|y_{n, i}-T_{i}\left(y_{n, i}\right)\right\|^{2}=0,
$$

which implies that $\lim _{n \rightarrow \infty}\left\|y_{n, i}-T_{i}\left(y_{n, i}\right)\right\|=0$ and $\lim _{n \rightarrow \infty}\left\|x_{n}-y_{n, i}\right\|=0(i \in \mathscr{I})$.

(iii) The same discussion as for the proof of Lemma 3.2(ii) leads to the assertion in Lemma 3.5(iii).

Lemma 3.5 leads to the following result.

Lemma 3.6. Suppose that Assumption 3.2 holds. Then, the following hold: 
(i) $\limsup _{n \rightarrow \infty} f\left(x_{n}\right) \leq f(x)(x \in X)$;

(ii) Any weak sequential cluster point of $\left(x_{n}\right)_{n \in \mathbb{N}}$ is in $X^{\star}$.

Proof. (i) The same discussion as for showing the existence of $N_{2}$ in the proof of Lemma 3.2(iii) ensures that there exists $M_{6} \in \mathbb{R}$ such that, for all $x \in X$ and for all $n \in \mathbb{N}$,

$$
f(x)-\sum_{i \in \mathscr{I}} f_{i}\left(y_{n, i}\right) \leq f(x)-f\left(x_{n}\right)+M_{6} \sum_{i \in \mathscr{I}}\left\|x_{n}-y_{n, i}\right\| .
$$

Hence, (3.14) guarantees that, for all $x \in X$ and for all $n \in \mathbb{N}$,

$$
\frac{2}{I}\left(f\left(x_{n}\right)-f(x)\right) \leq\left(\left\|x_{n}-x\right\|+\left\|x_{n+1}-x\right\|\right) \frac{\left\|x_{n+1}-x_{n}\right\|}{\gamma_{n}}+M_{4} \frac{\alpha_{n}}{\gamma_{n}}+M_{6} \sum_{i \in \mathscr{I}}\left\|x_{n}-y_{n, i}\right\|,
$$

which, together with (C3) and Lemma 3.5(i) and (ii), implies that $\limsup _{n \rightarrow \infty} f\left(x_{n}\right) \leq f(x)(x \in X)$.

(ii) Since $\left(x_{n}\right)_{n \in \mathbb{N}}$ is bounded, there exists a weak sequential cluster point of $\left(x_{n}\right)_{n \in \mathbb{N}}$. Let $x^{\star} \in H$ be an arbitrary weak sequential cluster point of $\left(x_{n}\right)_{n \in \mathbb{N}}$. Then, there exists $\left(x_{n_{k}}\right)_{k \in \mathbb{N}} \subset\left(x_{n}\right)_{n \in \mathbb{N}}$ which weakly converges to $x^{\star}$. The same discussion as for showing $x^{*} \in X$ in the proof of Lemma 3.2(iv), together with Lemma 3.5(iii), leads to $x^{\star} \in X$. Moreover, Lemma 3.6(i) and (A2) guarantee that, for all $x \in X$,

$$
f\left(x^{\star}\right) \leq \liminf _{k \rightarrow \infty} f\left(x_{n_{k}}\right) \leq \limsup _{k \rightarrow \infty} f\left(x_{n_{k}}\right) \leq \limsup _{n \rightarrow \infty} f\left(x_{n}\right) \leq f(x),
$$

which implies that $x^{\star} \in X^{\star}$. This completes the proof.

\section{CONCLUSION}

This paper presented two parallel proximal point algorithms for solving the problem of minimizing the sum of convex functions over the intersection of fixed point sets of nonexpansive mappings. One of the two parallel algorithms is based on the Krasnosel'skiî-Mann fixed point algorithm and the other one is based on the Halpern fixed point algorithm. We showed that there exists a subsequence of the sequence generated by the Krasnosel'skil-Mann type algorithm which weakly converges to a solution of the problem under certain assumptions. We also showed that any weak sequential cluster point of the sequence generated by the Halpern type algorithm belongs to the solution set of the problem.

\section{Acknowledgments}

The authors are sincerely grateful to the handling editor and the anonymous referees for helping us improve the original manuscript. This paper was supported by JSPS KAKENHI Grant Numbers JP15K04763 and JP18K11184.

\section{REFERENCES}

[1] H. H. Bauschke, P. L. Combettes, Convex Analysis and Monotone Operator Theory in Hilbert Spaces, Springer, New York, (2011)

[2] V. Berinde, Iterative Approximation of Fixed Points, Springer, Berlin, (2007)

[3] B. Halpern, Fixed points of nonexpanding maps, Bull. Amer. Math. Soc. 73 (1967), 957-961.

[4] H. Iiduka, Fixed point optimization algorithms for distributed optimization in networked systems, SIAM J. Optim. 23 (2013), 1-26.

[5] H. Iiduka, Parallel computing subgradient method for nonsmooth convex optimization over the intersection of fixed point sets of nonexpansive mappings, Fixed Point Theory Appl. 2015 (2015), Article ID 72. 
[6] H. Iiduka, Convergence analysis of iterative methods for nonsmooth convex optimization over fixed point sets of quasinonexpansive mappings, Math. Program. 159 (2016), 509-538.

[7] H. Iiduka, Incremental subgradient method for nonsmooth convex optimization with fixed point constraints, Optim. Methods Software 31 (2016), 931-951.

[8] H. Iiduka, Proximal point algorithms for nonsmooth convex optimization with fixed point constraints, Euro. J. Oper. Res. 253 (2016), 503-513.

[9] H. Iiduka, Distributed optimization for network resource allocation with nonsmooth utility functions, IEEE Trans. Control Network Sys. DOI: 10.1109/TCNS.2018.2889011.

[10] H. Iiduka, K. Hishinuma, Acceleration method combining broadcast and incremental distributed optimization algorithms, SIAM J. Optim. 24 (2014), 1840-1863.

[11] H. Iiduka, I. Yamada, Computational method for solving a stochastic linear-quadratic control problem given an unsolvable stochastic algebraic Riccati equation. SIAM J. Control Optim. 50 (2012), 2173-2192.

[12] M. A. Krasnosel'skiĭ, Two remarks on the method of successive approximations, Uspekhi Matematicheskikh Nauk 10 (1955), 123-127.

[13] P. E. Maingé, The viscosity approximation process for quasi-nonexpansive mappings in Hilbert spaces, Comput. Math. Appl. 59 (2010), 74-79.

[14] W. R. Mann, Mean value methods in iteration, Proc. Amer. Math. Soc. 4 (1953), 506-510.

[15] G. J. Minty, A theorem on maximal monotonic sets in Hilbert space, J. Math. Anal. Appl. 11 (1965), 434-439.

[16] J. J. Moreau, Fonctions convexes duales et points proximaux dans un espace hilbertien, C. R. Acad. Sci. Paris Sér. A Math. 255 (1962), 2897-2899.

[17] Z. Opial, Weak convergence of the sequence of successive approximation for nonexpansive mappings, Bull. Amer. Math. Soc. 73 (1967), 591-597.

[18] R. Wittmann, Approximation of fixed points of nonexpansive mappings, Arch. Math. 58 (1992), 486-491.

[19] I. Yamada, The hybrid steepest descent method for the variational inequality problem over the intersection of fixed point sets of nonexpansive mappings, In: D. Butnariu, Y. Censor, S. Reich (eds.) Inherently Parallel Algorithms for Feasibility and Optimization and Their Applications, Elsevier, New York (2001), 473-504. 